\title{
GPS Data Filtration Method for Drive Cycle Analysis Applications
}

\author{
Adam Duran and Matthew Earleywine \\ National Renewable Energy Laboratory
}

\begin{abstract}
Global Positioning System (GPS) data acquisition devices have proven useful tools for gathering real-world driving data and statistics. The data collected by these devices provide valuable information in studying driving habits and conditions. When used jointly with vehicle simulation software, the data are invaluable in analyzing vehicle fuel use and performance, aiding in the design of more advanced and efficient vehicle technologies. However, when employing GPS data acquisition systems to capture vehicle drive-cycle information, a number of errors often appear in the captured raw data samples. Common sources of error in GPS data include sudden signal loss, extraneous or outlying data points, speed drifting, and signal white noise, all of which combine to limit the quality of field data for use in downstream applications. Unaddressed, these errors significantly impact the reliability of source data and limit the effectiveness of traditional drive cycle analysis approaches and vehicle simulation software. Without reliable speed and time information, the validity of derived metrics for drive cycles, such as acceleration, power, and distance become questionable. This study explores some of the common sources of error present in collected raw GPS data and presents a detailed filtering process designed to correct for these issues. To illustrate the effectiveness of the proposed filtration process across the range of vehicle vocations, test data from both light- and medium/heavy-duty applications are examined. Graphical comparisons of raw and filtered cycles are presented, and statistical analyses performed to determine the effects of the proposed filtration process on raw data. Finally, the paper concludes with an evaluation of the overall benefits of data filtration on raw GPS data and presents potential areas for continued research.
\end{abstract}

\section{INTRODUCTION}

The cost-effective nature and ease of installation associated with GPS data acquisition systems (DASs) have aided in onboard global positioning system (GPS) data logging rapidly becoming one of the more popular methods for collecting real-world vehicle operating information $[\underline{1}, \underline{2}, \underline{3}$, 4]. The coupled vehicle speed-time data captured by these devices are of particular interest when performing vehicle simulation and drive cycle analysis $[\underline{5}, \underline{6}, \underline{7}, \underline{8}, \underline{9}]$. However, due to the sensitivity of these downstream applications to both the quality and integrity of GPS source data, the unique operating behavior and errors inherently associated with GPS data loggers have fostered a need for a novel filtration process. The ideal filtration method for improving the quality of raw GPS data is one that minimizes the effects of GPS data logging errors such as sudden signal loss, data spiking, signal white noise, and zero speed drift while maintaining the integrity of the raw source data $[\underline{10}, \underline{11}, \underline{12}]$. This means removing or replacing any erroneous data points while simultaneously minimizing the total amount of data altered. The ideal filtration method must also be flexible enough for application to GPS data sourced from different logging devices. As such, any proposed filtration method must take into account differences in data quality available through different GPS DASs as well as the different vehicle vocations or types in which the loggers may be mounted.

The GPS data filtration approach presented here consists of seven distinct filters designed and arranged specifically to meet these criteria. In an attempt to account for the common errors associated with the analysis of GPS speed-time data, a common GPS data processing method has been developed in the Matlab environment that employs a series of linearly progressing logic-based data filters [1] $]$. 
To evaluate the effectiveness of the proposed GPS data filtration process, National Renewable Energy Laboratory (NREL) researchers analyzed over 1,700 individual drive cycles sourced from GPS devices mounted onboard both light- and medium/heavy-duty vehicles. The drive cycles analyzed as part of this study contain coupled speed-time information gathered using onboard GPS DASs, and contain the driving information for either a single vehicle operating day or shift. Statistics regarding the amount of data altered during each filtration step were calculated, and the results of the filtration process were analyzed. In addition to examining the magnitude of data altered during the filtration process, drive-cycle analysis calculations were performed to assess the effects of filtration on the underlying cycle dynamics.

\section{APPROACH}

\section{FILTER LOGIC}

The proposed filtration process for analyzing GPS speed-time data for drive-cycle applications consists of seven logic-based filters arranged in order of increasing complexity. They are as follows:

1. Remove duplicate records and data with negative values or differential time steps

2. Replace outlying high/low speed values

3. Remove zero-speed signal drift when vehicle is stopped

4. Replace false zero-speed records

5. Amend gaps in data

6. Repair outlying acceleration/deceleration values

7. Denoise and condition final signal.

Removing Data with Duplicate/Negative Time $\underline{\text { Records }}$

As an initial step in the filtration process, it is necessary to remove any data points with duplicate time values and data points that have negative or zero differential time values (i.e., time at point 2 is less than time at point 1 , or time at point 2 is equal to time at point 1 ). To remove these points, the filter first calculates the differential time values for each of the data points in the source set and then removes any with differential time values less than or equal to zero.

This step must be performed first due to the influence of differential time when calculating acceleration from speed information. If duplicate time records exist, then by definition of acceleration from basic kinematics, the acceleration at the duplicate point would approach infinity. If erroneous time information were not removed in this preliminary step, we would be unable to perform accurate calculations based on differential/integral time information, and the future filtration steps that rely on this information would be impossible.

\section{$\underline{\text { Replacing Outlying Speed Values }}$}

In the second step in the filtration process, any erroneous data points, such as single-sample high-speed data spikes and negative speed signal dropouts, that are present in the data set are removed and replaced with interpolated data. To achieve this, each speed value in the sample set is processed through the filter and compared individually to chosen high/low speed limits. If the data point is found to lie outside the chosen limits, the filter replaces the source sample data point with speed information derived from a cubic spline interpolation drawn from neighboring data [14]. As an added step in the interpolation process, the filtered vehicle speed is limited on the lower bound to zero miles per hour to facilitate vehicle simulation and dynamometer testing. The application of usercontrolled boundary limits allows for flexibility during data processing and improves the quality of the results when vehicle speed limitations are known.

In Figure 1, a 75-mph high-speed limit was used in conjunction with the outlying-speed filter to remove the erroneous high-speed signal spike in the sample GPS vehicle data. The limit was applied assuming that speeds in excess of $75 \mathrm{mph}$ could be attributed to signal spikes and not normal operation. For the case of unknown vehicles, selecting more conservative high/low operating limits is advised to avoid removal of accurate information. Employing controllable limits in the outlying-speed filter provides a unique advantage when filtering large numbers of drive cycles as filtration limits can be adjusted for individual vehicle types.

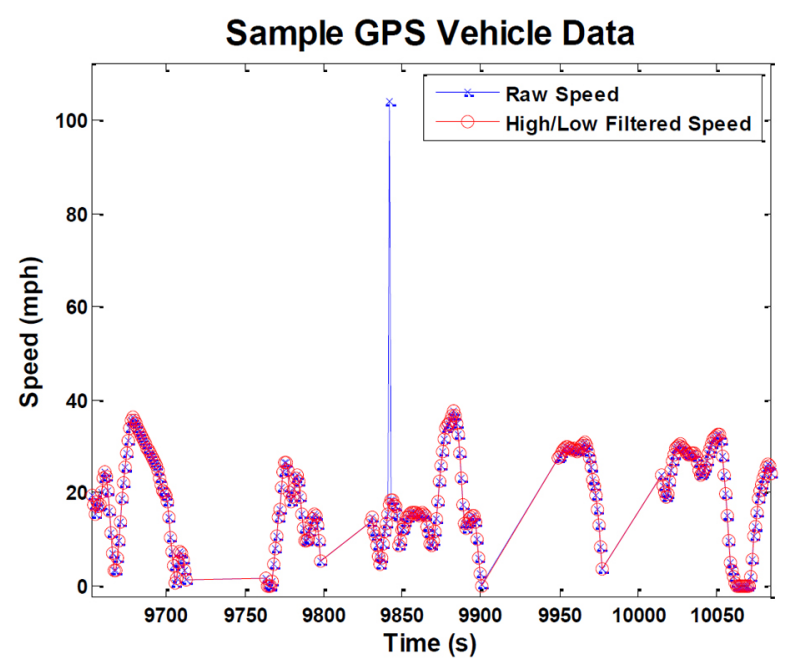

Figure 1. Outlying speed filtration results for sample GPS vehicle data.

\section{Removing GPS Zero-Speed Drift}

In the third step of the filtration process, an effect called "zero speed drift" is removed. Zero speed drift appears when a GPS DAS is running and the vehicle is stopped and idling for a prolonged period of time. During extended-duration idle 
events, GPS data loggers will often record a very small speed value $(0.1$ or $0.2 \mathrm{mph})$ due to GPS satellite signal reacquisition that occurs when a vehicle is stopped. To remove the small fluctuations in vehicle speed recorded by the GPS DAS during these periods, the zero-speed drift filter examines the distance traveled during each microtrip in a drive cycle and compares the value with a user-specified limit. If the distance traveled over the course of the microtrip is below the limit, the entire microtrip is replaced with zerospeed data values.

As seen in Figure 2, applying a 0.001-mile distance limit to the sample GPS data causes removal of the low-speed microtrip. When performing traditional integration-based distance calculations using coupled speed-time information, removing the zero-speed drift data results in lower calculated distances, which are more reflective of actual vehicle operation. Similarly, when performing simulations using plug-in hybrid-electric and electric vehicle (PHEV and EV) models, removing these zero-speed drift segments results in more accurate energy consumption calculations and improved state-of-charge estimates.

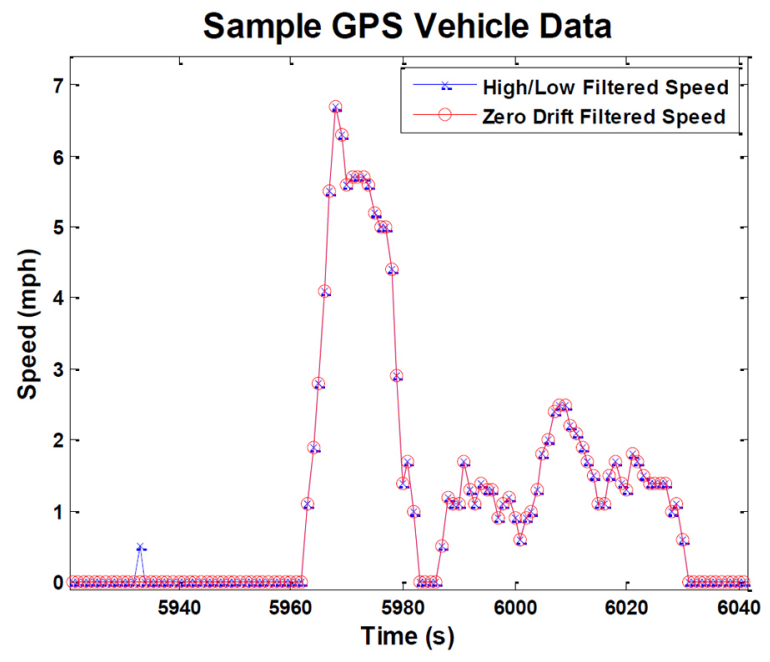

Figure 2. Zero-speed drift filter results for sample GPS vehicle data.

\section{$\underline{\text { Replacing False Zero-Speed Records }}$}

The next step in the filtration process, the false zero-speed filter, removes single-point zero-speed data records that are the result of temporary GPS signal dropout. Similar to the outlying speed filter, the false-zero filter removes false zerospeed records by examining the value of each individual speed point in the data set in relation to its nearest neighbors. If a given speed record value is zero and the neighboring points on each side are both nonzero, the zero-speed point is replaced with a point drawn from a cubic spline interpolation of the entire remaining data set.
This simple filter, shown in Figure 3 , improves the continuity of the raw data by replacing significant outliers in the data with data more representative of true vehicle operation. By removing the dramatic changes in speed, energy calculations are improved when analyzing the drive cycle; more importantly, in vehicle simulation scenarios the model is capable of accurately following the prescribed speed-time trace.

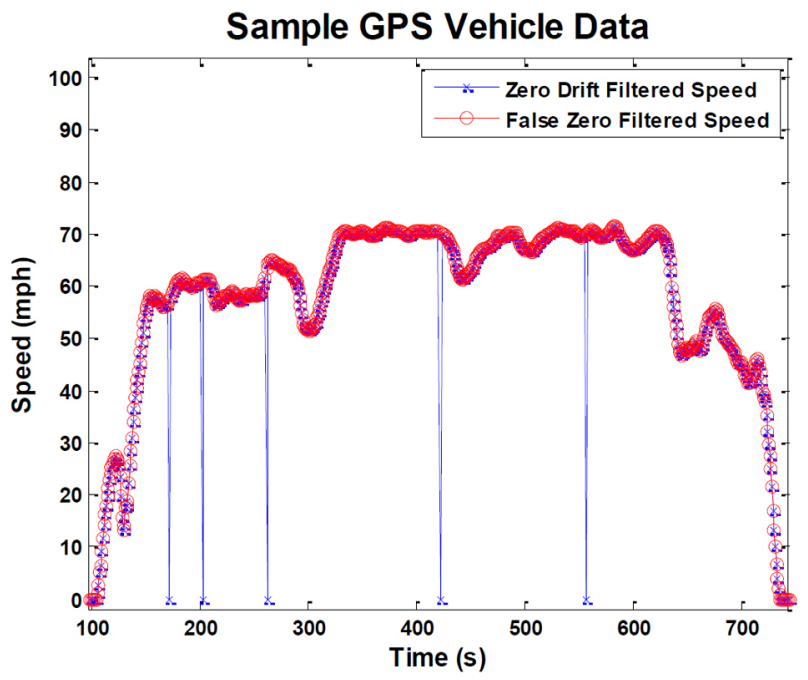

Figure 3. False zero-speed filter results for sample GPS vehicle data.

\section{Amending Signal Gaps}

In this step, the filtration algorithm attempts to correct for gaps in the coupled speed-time GPS signal caused by urban canyon effects and sudden signal loss. Employing a usersupplied time gap limit, the filter examines the time stamp information pulled from the input GPS data stream and attempts to interpolate over any signal gaps that are shorter in duration than the specified limit. If the signal gap duration is shorter than the limit, the algorithm generates monotonically increasing time signals based on the sampling rate of the underlying source data. To generate the "new" speed data, the same interpolated cubic spline curve fit used in previous filtration steps is applied over the newly generated time domain.

If the filter detects a time gap greater than the user-defined limit, as shown in Figure 4, the filter ramps the vehicle speed down or up based on whether or not the data point is at the leading or trailing boundary of the gap in the signal. The ramp is constructing based on user-specified acceleration/ deceleration limits. Construction of the ramps is particularly important when attempting to adapt field data for chassis dynamometer and vehicle simulation applications. Fixing time gaps is the only step in the filtration process that will add data to the existing GPS data set (coupled time-speed information). Along with testing/experimental applications, 
the signal reconstruction provided by the signal gap filter is also important on an empirical level when examining the duty cycle of the vehicle, especially when performing analysis on real-time operation. Accurate knowledge of vehicle key on/off time is integral in determining the potential for opportunity charging events for PHEVs or EVs during daily operation, as well as cool-down times for power electronics and other thermal components.

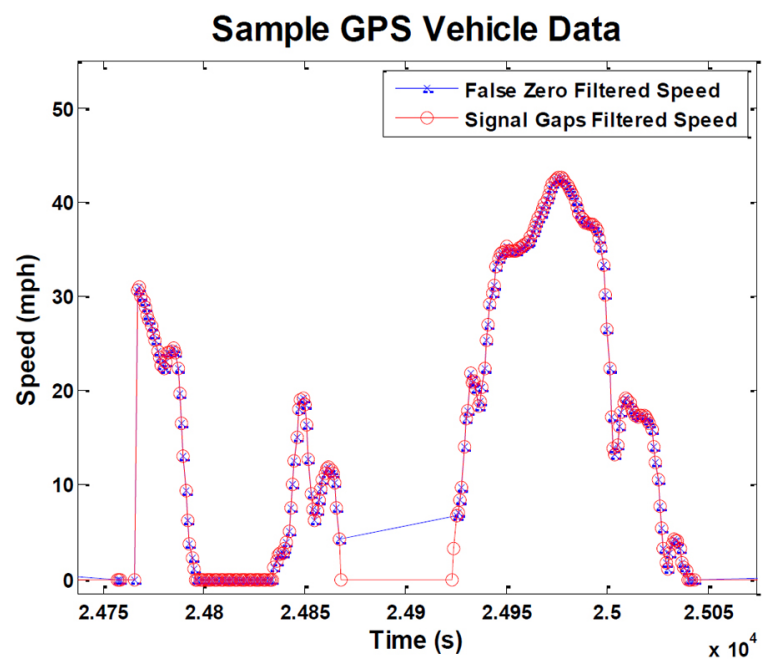

Figure 4. Signal gap filter results for sample GPS vehicle data.

\section{Repairing Outlying Acceleration/Deceleration}

\section{Values}

In the last major step in the filtration process, an algorithm attempts to fix outlying accelerations/decelerations that remain in the raw GPS data set after all prior filtration steps. While the outlying speed filter removed speed points that existed outside the realm of velocity limits, this filter looks at the derivatives with respect to time of the speed data to see if the recorded data matches expected vehicle performance levels. The filter loops through the data set and progressively refines and smoothes the acceleration of outlying points. While looping through the data set, if the acceleration/ deceleration of a data point is outside the user-supplied limits, the point is replaced with a value that is calculated using the interpolation method described above. However, if the interpolated value still produces an acceleration outside of the acceptable limits, the filter adjusts the new data point to fit within $1 \%$ of the user-defined limits $(+1 \%$ for acceleration, $-1 \%$ for deceleration). This filter loops through the speed/ time information until all acceleration/deceleration values fall within the user-defined limits (Figure 5).

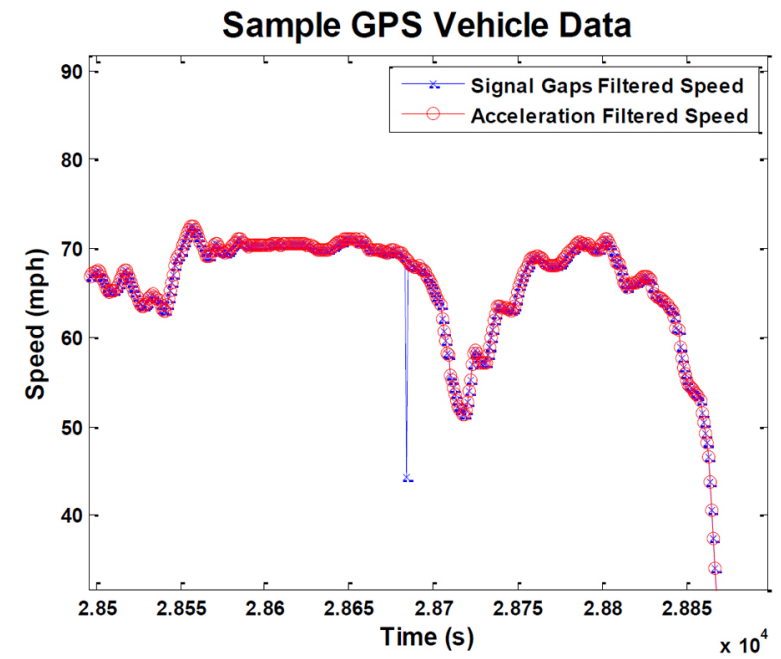

Figure 5. Outlying acceleration filter results for sample GPS vehicle data.

It is important to select limits for this filter that closely match the performance expected of the vehicle. Applying heavyduty acceleration/deceleration limits to a light-duty vehicle would result in large amounts of original data being removed; similarly, applying light-duty limits to heavy-duty vehicle data would remove too little data. In cases where vehicle and power train information are known, a rough estimate of appropriate acceleration/deceleration limits can be determined. Otherwise, conservative limits based on vehicle classification and weight are recommended.

\section{Denoising and Conditioning the Signal}

The final step in the filtration process combines a localized least squares polynomial smoothing filter [commonly known

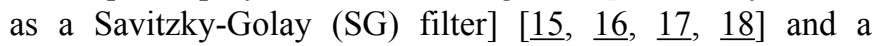
binomial smoothing filter $[\underline{19}-\underline{20}]$. The combined SGbinomial filter reduces the effect of signal white noise while maintaining the overall profile of the drive cycle. The advantage of the combined SG-binomial filter is that the SG component acts as a complementary form of the acceleration/ deceleration filter by definition minimizing the effects of any outlying artifacts remaining from prior filtration steps, while the binomial component of the filter acts to remove any underlying white noise found in the signal. As shown in Figure 6, the filter is able to maintain the shape of the original drive cycle while removing any remaining outliers and background signal noise. 


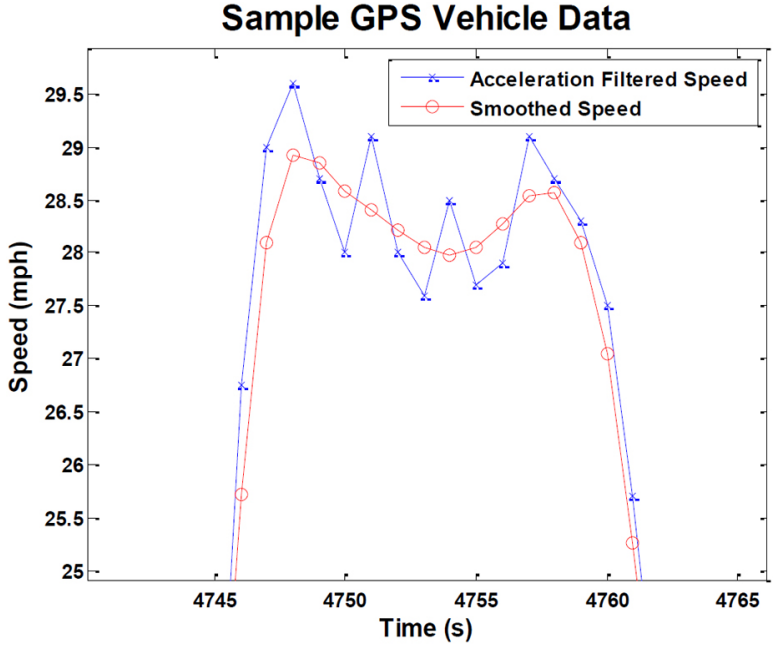

Figure 6. Denoising filter applied to time-speed GPS vehicle data.

Removing noise from raw GPS data signals and providing additional smoothing conditions are integral in preparing source data for use in chassis dynamometer/vehicle simulation applications. For example, removing existing signal transients and background noise is especially important when running long-duration chassis dynamometer tests. Instead of having testing personnel attempt to follow a highly variable speed-time trace due to signal noise components, the testing personnel are able to maintain more natural driving behavior and follow a smooth driving profile more in line with typical driving behavior. For vehicle simulation activities, removing the remaining signal noise avoids complications that arise when simulating advanced vehicle (PHEV/EV) control strategies. Noisy speed profiles generate incorrect power demand information, resulting in inaccurate state-of-charge and energy consumption calculations, directly correlating to reduced simulated fuel economy values.

\section{GPS DATA COLLECTION}

To evaluate the effectiveness of the proposed filtration process, the authors analyzed two unique sets of GPS speedtime data. The light-duty data set was drawn from a collection of unknown light-duty vehicles [21] instrumented with GeoStats GeoLoggers as part of the year 2000 Southern California Association of Governments post-census regional travel survey, while the heavy-duty data set was drawn from class 3 to class 8 heavy-duty vehicle data collected from 2008 to present $[\underline{7}, \underline{22}, 23]$. The GPS data for the heavy-duty data set was collected using a combination of GeoStats GeoLoggers and Isaac Instruments DRU908 and DRU900 data acquisition devices. The light-duty data set contained 1,202 individual drive cycles, while the heavy-duty set contained 588 individual drive cycles. The goal of selecting two unique data sets, one light duty and the other heavy duty, consisting of a mix of known and unknown vehicles, was to evaluate the effectiveness of the filtration process across a wide range of vehicle types and vocations.

\section{FILTER INPUTS}

Before evaluating the effectiveness of the proposed filtration process, acceptable inputs for each stage in the filtration process were established. Conservative limits were selected for both the light- and heavy-duty vehicle data sets, with the focus on setting speed and acceleration limits at the bounds of realistic operation. For the light-duty vehicle data set, a vehicle speed range of up to $120 \mathrm{mph}$ and maximum absolute acceleration limits of approximately $\pm 0.8 \mathrm{G}$ were selected. These values were chosen based on the performance characteristics of a 2010 Chevy Corvette, the maximum performance values likely in a light-duty vehicle [24]. For the zero-speed drift filter and the signal gap filter, standard values were selected based on GPS signal reacquisition technical specifications for a Garmin 18x GPS receiver [25], and an assigned minimum trip distance of approximately 50 $\mathrm{ft}$. Table 1 summarizes the filtration inputs for the light-duty vehicle data set.

\section{Table 1. Light Duty Vehicle Data Set Filtration Limits}

\begin{tabular}{|c|c|}
\hline Filter Name & Filter Ranges \\
\hline Negative/Duplicate & N/A \\
\hline Zero Speed Drift & $0.01 \mathrm{mi}$ \\
\hline False Zero Speed & N/A \\
\hline Signal Gaps Filter & $15 \mathrm{~s}$ \\
\hline Acceleration Filter & -17.5 to $+17.5 \mathrm{mph} / \mathrm{s}$ \\
\hline
\end{tabular}

For the heavy-duty vehicle data set, the acceleration range limits were selected based on a drive-cycle analysis of standard heavy-duty chassis dynamometer test cycles. The Rowan University Composite School Bus Cycle (RUCSBC) [26] contained the greatest acceleration rate of the 32 standard cycles examined; therefore, the maximum acceleration in the RUCSBC cycle was chosen as the limiting range for the acceleration filter. The distance and time-gap limits for the heavy-duty vehicle data set were chosen to match those of the light-duty vehicle data set.

Table 2. Heavy Duty Data Set Filtration Limits

\begin{tabular}{|c|c|}
\hline Filter Name & Filter Ranges \\
\hline Negative/Duplicate & N/A \\
\hline High/Low Speed & $0-80 \mathrm{mph}$ \\
\hline Zero Speed Drift & $0.01 \mathrm{mi}$ \\
\hline False Zero Speed & N/A \\
\hline Signal Gaps Filter & $15 \mathrm{~s}$ \\
\hline Acceleration Filter & -8.8 to $+8.8 \mathrm{mph} / \mathrm{s}$ \\
\hline
\end{tabular}


Table 3. Light-Duty Vehicle Filtration Results

\begin{tabular}{|c|c|c|c|}
\hline Filter Name & Mean (\%) & Median (\%) & $\boldsymbol{\sigma ( \% )}$ \\
\hline Negative/Duplicate & 3.065 & 0.326 & 5.254 \\
\hline High/Low Speed & 0.004 & 0 & 0.017 \\
\hline Zero Speed Drift & 0.311 & 0.192 & 0.591 \\
\hline False Zero Speed & 0.179 & 0.132 & 0.223 \\
\hline Signal Gaps Filter & 19.550 & 18.044 & 11.151 \\
\hline Acceleration Filter & 0.557 & 0.243 & 1.584 \\
\hline
\end{tabular}

Table 4. Light-Duty Drive Cycle Metrics

\begin{tabular}{|c|c|c|c|c|c|c|}
\hline & \multicolumn{3}{|c|}{ RAW } & \multicolumn{3}{c|}{ FILTERED } \\
\cline { 2 - 7 } & Median & Mean & $\boldsymbol{\sigma}$ & Median & Mean & $\boldsymbol{\sigma}$ \\
\hline Maximum Driving Speed (mph) & 76.01 & $40,158.70$ & $645,940.50$ & 71.35 & 67.11 & 15.20 \\
\hline Mean Cycle Speed (mph) & 26.45 & 32.55 & 73.73 & 26.33 & 28.17 & 9.64 \\
\hline Mean Driving Speed (mph) & 30.65 & 36.53 & 75.39 & 29.95 & 31.51 & 9.42 \\
\hline Standard Deviation Speed (mph) & 19.85 & 424.47 & $6,739.95$ & 19.11 & 19.29 & 4.62 \\
\hline Zero Speed Time (\%) & 12.08 & 12.41 & 5.48 & 11.25 & 11.64 & 4.99 \\
\hline Characteristic Acceleration (ft/s/s) & 0.27 & $17,538.07$ & $373,062.12$ & 0.63 & 0.64 & 0.19 \\
\hline Aerodynamic Speed (ft/s) & 49.29 & $31,025.36$ & $585,366.40$ & 64.42 & 65.16 & 17.00 \\
\hline Kinetic Intensity (1/mile) & 0.41 & 0.77 & 1.05 & 0.84 & 1.11 & 0.95 \\
\hline
\end{tabular}

\section{RESULTS}

\section{LIGHT DUTY}

The filtration results for the light-duty vehicle data set are shown in Table 3. One first observes that on average approximately $20 \%$ of the original data set is undergoing some level of filtration. This would appear to be a high value; however, when the two filtration steps that add/remove data based on signal discontinuity are excluded, we see that, on average, less than $1 \%$ of the collected data are undergoing any filtration. These results support the goal of minimizing the total amount of signal processing performed on raw data; however, they raise questions about the quality of the source GPS data. The approximately $20 \%$ addition/removal of data that is occurring during the negative/duplicate time and signal gaps filter suggest source data with poor continuity and significant data acquisition issues.

Comparing the raw and filtered data for the light-duty vehicle GPS drive-cycle samples, shown in Table 4, we can see that outlying speed data in the raw data set generate dramatic effects on the calculated mean maximum driving speed, characteristic acceleration, and aerodynamic speed [4]. While only $0.004 \%$ of data are adjusted on average as part of the high/low speed filter, the outlying speeds in the raw data set prior to this filtration step render global drive-cycle statistics calculated from the raw data useless for direct comparison. Due to the existence of these outliers, the average maximum driving speed of the raw drive cycles is three orders of magnitude higher than that of the filtered data.

Performing paired t-tests to determine the effects of the filtration on the drive-cycle metrics, it was found that the proposed filtration process produced statistically significant changes in the mean values for each of the drive-cycle metrics examined with the exception of characteristic acceleration and aerodynamic speed. The large standard deviations for the raw data set contribute to the uncertainty in statistically determining the effects of the filtration on characteristic acceleration and aerodynamic speed; however, visually examining the data reveals the effects of filtration to be nontrivial.

\section{HEAVY DUTY}

Examining the filtered results for the heavy-duty vehicle data set, shown in Table 5, one observes that compared to the approximate $20 \%$ data filtration for the light-duty vehicle data, the heavy duty vehicle data examined in the study showed less than $5 \%$ filtration on average. When the two filtration steps that add/remove data are ignored, less than $0.5 \%$ of the raw data collected undergoes filtration on average.

Performing drive-cycle analysis comparisons on both the raw and filtered heavy-duty vehicle GPS drive-cycle samples as shown in Table 6 , outlying data appear to contribute a 
Table 5. Heavy Duty Filtration Results

\begin{tabular}{|c|c|c|c|}
\hline Filter Name & Mean (\%) & Median (\%) & $\boldsymbol{\sigma ( \% )}$ \\
\hline Negative/Duplicate & 0.069 & 0 & 0.285 \\
\hline High/Low Speed & 0 & 0 & 0 \\
\hline Zero Speed Drift & 0.098 & 0.067 & 0.114 \\
\hline False Zero Speed & 0.050 & 0.038 & 0.060 \\
\hline Signal Gaps Filter & 4.359 & 3.716 & 2.676 \\
\hline Acceleration Filter & 0.011 & 0.000 & 0.118 \\
\hline
\end{tabular}

Table 6. Heavy Duty Drive Cycle Metrics

\begin{tabular}{|c|c|c|c|c|c|c|}
\hline & \multicolumn{3}{|c|}{ RAW } & \multicolumn{3}{c|}{ FILTERED } \\
\cline { 2 - 7 } & Median & Mean & $\boldsymbol{\sigma}$ & Median & Mean & $\boldsymbol{\sigma}$ \\
\hline Maximum Driving Speed (mph) & 61.65 & 58.43 & 10.09 & 61.19 & 58.04 & 10.06 \\
\hline Mean Cycle Speed (mph) & 13.06 & 13.50 & 5.14 & 13.21 & 13.62 & 5.06 \\
\hline Mean Driving Speed (mph) & 25.10 & 25.75 & 4.85 & 24.53 & 25.19 & 4.82 \\
\hline Standard Deviation Speed (mph) & 15.82 & 15.99 & 3.18 & 15.82 & 15.95 & 3.14 \\
\hline Zero Speed Time (\%) & 46.11 & 48.29 & 14.47 & 44.37 & 46.63 & 14.44 \\
\hline Characteristic Acceleration (ft/s/s) & 0.55 & 0.55 & 0.09 & 0.50 & 0.50 & 0.08 \\
\hline Aerodynamic Speed (ft/s) & 51.28 & 52.30 & 9.31 & 51.90 & 52.80 & 9.24 \\
\hline Kinetic Intensity (1/mile) & 1.14 & 1.36 & 3.95 & 1.02 & 1.16 & 2.27 \\
\hline
\end{tabular}

minimal effect on the quality of the raw heavy-duty vehicle data when compared to the light-duty vehicle data. On average, the maximum and average driving speed of the cycle are reduced, while the total speed of the cycle increases, suggesting the repair of signal dropouts and spikes.

Performing paired t-tests to determine the effects of the filtration on the drive-cycle metrics, it was found that the proposed filtration process produced statistically significant changes in the mean values for each of the drive-cycle metrics examined. However, examining the differences in the means, medians, and standard deviations of the metrics examined, it appears that while the changes in mean are statistically significant, overall the change in the data are minimal on the whole.

\section{CONCLUSION}

Based on the results of this study, it can be concluded that the proposed GPS data filtration adequately fulfills the requirements proposed for an ideal GPS data filter. Excluding removal of erroneous data and addition of lost signal components, the proposed filtration process minimally affects the source data while simultaneously generating significant improvements in the quality of data required for calculation of drive-cycle metrics used as part of drive-cycle analysis and vehicle simulation applications.
Analyzing the filtered results from both the light- and heavyduty vehicle data sets, gaps in signal acquisition and underlying data acquisition issues are a much greater component of the filtration process than previously anticipated. The loss of GPS signal is the greatest factor contributing to the loss in data quality on a point-per-point basis, accounting for at least an order of magnitude greater influence in the filtration process than the other sources of error. However, examining the duty-cycle analysis results based on the filtered and raw data for both the light- and heavy-duty vehicle data sets, it was also shown that removing data spikes is the key step in producing accurate vehicle speed/acceleration estimates.

Based on the results of this study coupled with increased interest in the integration of elevation/road grade information into engineering analyses, in the future it is recommended that additional filtration methods be developed to repair collected GPS elevation information. Combining an additional dimension to the filtration process should introduce additional logic-based filtration opportunities that do not currently exist when examining the data in a single dimension. 


\section{REFERENCES}

1. Ivani, Ž., "Data Collection and Development of New York City Refuse Truck Duty Cycle." SAE Technical Paper 2007-01-4118, 2007, doi:10.4271/2007-01-4118.

2. Casey, E.J., Smith, W.J., and Timoney, D.J., "Examination of Low-cost Systems for the Determination of Kinematic Driving Cycles and Engine Operating Conditions in Dublin, Ireland," SAE Technical Paper 2009-01-2791, 2009, doi:10.4271/2009-01-2791.

3. Dembski, N., Rizzoni, G., Soliman, Fravert, J. et al., "Development of Refuse Vehicle Driving and Duty Cycles," SAE Technical Paper 2005-01-1165, 2005, doi: 10.4271/2005-01-1165.

4. O'Keefe, M.P., Simpson, A., Kelly, K.J., and Pedersen, D.S., "Duty Cycle Characterization and Evaluation Towards Heavy Hybrid Vehicle Applications," SAE Technical Paper 2007-01-0302, 2007, doi:10.4271/2007-01-0302.

5. Frey, H.C., et al. "In-Use Measurement of the Activity, Energy Use, and Emissions of a Plug-in Hybrid Electric Vehicle," Paper 2009-A-242-AWMA, Proceedings, 102nd Annual Conference and Exhibition, Air \& Waste Management Association, Detroit, Michigan, June 16-19 2009.

6. Earleywine, M., et al. "Simulated Fuel Economy and Performance of Advanced Hybrid Electric and Plug-In Hybrid Electric Vehicles Using In-Use Travel Profiles." Vehicle Power and Propulsion Conference (VPPC), 2010 IEEE, pp 1-6, 1-3 September 2010.

7. Barnitt, R.A. and Gonder, J., "Drive Cycle Analysis, Measurement of Emissions and Fuel Consumption of a PHEV School Bus," SAE Technical Paper 2011-01-0863, 2011, doi:10.4271/2011-01-0863.

8. Kulkarni, A., Sapre, R.R., and Sonchal, C.P., "GPS Based Methodology for Drive Cycle Determination," SAE Technical Paper 2005-01-1060, 2005, doi: 10.4271/2005-01-1060.

9. Gonder, J., et al. "Using Global Positioning System Travel Data to Assess Real-World Energy Use of Plug-In Hybrid Electric Vehicles." Transportation Research Record: Journal of the Transportation Research Board, volume 2017, pp. 26-322007.

10. Witte, T.H., et al. "Accuracy of WAAS-enabled GPS for the determination of position and speed over ground." Journal of Biomechanics, Volume 38, Issue 8, pp. 1717-1722, August 2005.

11. Arnold, L., et al. "Positional accuracy of the Wide Area Augmentation System in consumer-grade GPS units." Computers \& Geosciences, Volume 37, Issue 7, pp. 883-892, July 2011.

12. Jackson, E., et al. "The Accuracy of GPS-Based Acceleration for Vehicle Emissions Modeling."
Transportation Research Board $86^{\text {th }}$ Annual Meeting, Paper No. 07-2202, 2007.

13. NREL Vehicle Drive Cycle Tool, User Guide. Copyright (C) 2009 Alliance for Sustainable Energy, LLC. All Rights Reserved.

14. Feng, G., "Data Smoothing by Cubic Spline Filters," Signal Processing, IEEE Transactions on, Volume 46, Issue 10, pp. 2790-2796, Oct 1998.

15. Savitzky, A., et al. "Smoothing and Differentiation of Data by Simplified Least Squares Procedures." Analytical Chemistry, Volume 36, Issue 8, pp. 1627-1639, 1964.

16. Madden, H., "Comments on the Savitzky-Golay Convolution Method for Least-Squares Fit Smoothing and Differentiation of Digital Data." Analytical Chemistry, Volume 50, Issue 9, pp. 1383-1386, 1978.

17. Bromba, M., et al. "Application Hints for Savitzky-Golay Digital Smoothing Filters." Analytical Chemistry, Volume 53, Issue 11, pp. 1583-1576, 1981.

18. Gorry, A., et al. "General Least-Squares Smoothing and Differentiation by the Convolution (Savitzky-Golay) Method." Analytical Chemistry, Volume 62, Issue 6, pp. 570-573, 1990.

19. Marchand, P., et al. "Binomial Smoothing Filter: A Way to Avoid Some Pitfalls Of Least-Squares Polynomial Smoothing." Review of Scientific Instruments, Volume 54, Issue 8, pp. 1034-1041, Aug 1983.

20. Aubury, M., et al. "Binomial Filters." Journal of VLSI Signal Processing, Volume 12, Issue 1, pp. 35-50, 1996.

21. NuStats, "Year 2000 Post-Census Regional Travel Survey, Final Report of Survey Results." Southern California Association of Governments, 2003.

22. Barnitt, R. "FedEx Express Gasoline Hybrid Electric Delivery Truck Evaluation: 12-Month Report.” Report TP-5400-48896, 2011.

23. Lammert, M. "Twelve-Month Evaluation of UPS Diesel Hybrid Electric Delivery Vans.” Report TP-540-44134, 2009.

24. Ponticel, P. "Focused on Fuel Economy." Automotive Engineering International, August 6 2010, pp. 28-30.

25. Garmin, "GPS 18x Technical Specifications." October 2011.pp. 6

26. Hearn, J., Toback, A., Akers, J., Hesketh, R.P. et al., "Development of a New Composite School Bus Test cycle and the Effect of Fuel Type on Mobile Emissions from Three School Buses," SAE Technical Paper 2005-01-1616, 2005, doi:10.4271/2005-01-1616.

\section{CONTACT INFORMATION}

Adam Duran is a research engineer working with the Center for Transportation Technologies and Systems at the National 
Renewable Energy Laboratory. Adam's work focuses primarily in the areas of drive cycle analysis and characterization, custom drive cycle development, and medium/heavy duty fleet evaluations. He may be reached at Adam.Duran@nrel.gov.

Matthew Earleywine is a research engineer working with the Center for Transportation Technologies and Systems at the National Renewable Energy Laboratory. His work focuses primarily in the areas of advanced vehicle modeling, simulation, \& analysis, vehicle charging infrastructure, and electric roadway implementation. He may be reached at Matthew.Earleywine@nrel.gov

\section{ACKNOWLEDGMENTS}

The authors wish to thank Lee Slezak, U.S. Department of Energy Vehicle Technologies Program, as well as the Department of Energy for its support in conducting this project. In addition, the support of NREL staff Ben Rosen, Jeff Gonder, and Kevin Walkowicz was invaluable to the completion of this work.

\section{DEFINITIONS/ABBREVIATIONS}

\section{DAS}

data acquisition system

EV

electric vehicle

$\mathbf{G}$

gravitational constant $\left(9.81 \mathrm{~m} / \mathrm{s}^{2}\right)$

GPS

global positioning system

\section{PHEV}

plug-in hybrid vehicle mph

mile per hour

NREL

National Renewable Energy Laboratory

\section{RUCSBC}

Rowan University Composite School Bus Cycle

$\mathbf{S}$

second

SG

Savitzky-Golay
The Engineering Meetings Board has approved this paper for publication. It has successfully completed SAE's peer review process under the supervision of the session organizer. This process requires a minimum of three (3) reviews by industry experts.

ISSN 0148-7191
Positions and opinions advanced in this paper are those of the author(s) and not necessarily those of SAE. The author is solely responsible for the content of the paper.

SAE Customer Service:

Tel: 877-606-7323 (inside USA and Canada)

Tel: 724-776-4970 (outside USA)

Fax: 724-776-0790

Email: CustomerService@sae.org

SAE Web Address: http://www.sae.org

Printed in USA 